\title{
Health promoting behaviour among Adolescents attending Adolescent friendly services at school campus.
}

\author{
${ }^{1}$ Shanthi Ramasubramaniam, ${ }^{2}$ Dr.Subhadra Iyengar \\ ${ }^{1}$ (Phd Scholar Asia E University, Study Center, Madurai) \\ ${ }_{2}^{2}$ (HOD department of research methods P.S.G College of Nursing, Coimbatore)
}

\begin{abstract}
Introduction: Adolescent health is often overseen in developing countries. Many needs of adolescents are left unmet. Adolescent Friendly Health Services (AFHS) which provide a broad range of preventive, promotive and curative services under one roof can help to ensure changes in health promoting behavior of adolescents. School is the ideal place to educate adolescents on health promotion. Objectives of the study:1.)To identify the health and development problems prevailing among the young adolescents. 2.) To determine the health promoting behaviours of adolescents who attended adolescent friendly services at school.3.)To provide guidance and counselling to adolescents on Physical health, psychosocial health, life style Modification and school performance through adolescent friendly centre. Research method: One group pretest -posttest design. Sample and setting: Total of 259 adolescents (158 girls and 101 boys) studying in the $9^{\text {th }}$ and $11^{\text {th }}$ standard of a private higher secondary school in Coimbatore district. Data analysis and interpretation: The data was analysed using SPSS 16 version. The main results of the study showed the "self "and "school" domains of the MSLSS scale had significant difference at 0.01 level before and after attending adolescent friendly services. Conclusion: Attending adolescent friendly services at school campus can bring about changes in health promoting behavior of adolescents.
\end{abstract}

Keywords: Adolescents, adolescent friendly services, guidance and counseling, health promotion, school. Abbreviations used: AFHS- Adolescent Friendly Health Services, WHO-World Health Organisation, MSLSSMulti Dimensional student life satisfaction Scale, BMI- Body Mass index,

\section{INTRODUCTION}

A huge proportion of the world's population - more than 1.75 billion is young, aged between 10 and 24 years. Adolescents (aged 10 to 19 years) have specific health and development needs, and many face challenges that hinder their well being, including poverty, a lack of access to health information and services, and unsafe environments. Interventions that address their needs can save lives and foster a new generation of productive adults who can help their communities' progress. Many boys and girls in developing countries enter adolescence undernourished, making them more vulnerable to disease and early death. Conversely, overweight and obesity another form of malnutrition with serious health consequences - is increasing among other young people in both low- and high-income countries. Adequate nutrition and healthy eating and physical exercise habits at this age are foundations for good health in adulthood. ( WHO Facts on Adolescent Health 2008). Viner R etal., 2005 in a clinical review on health promotion in adolescence has suggested that health promotion strategies and interventions can be delivered at various levels including individual, family, school, community and national levels. The interventions at school level can be single issue, curriculum based education and "whole school" organisation and behavior management interventions.

In India, health promotion is now receiving an increasing attention regarding the prominent role it plays in health. Health promoting lifestyle is one factor that positively contributes to quality of life. When a person engages in health promoting lifestyle, he/she has a greater potential to remain healthy and possibly live longer without the burden of the disease ( Raj S,Senjam et al., 2012).

Promoting health through schools is a 'life-course' approach to promote healthy behaviour among children. Many of today's and tomorrow's leading causes of death, disease and disability can be significantly reduced by preventing behaviour that is initiated during youth, through health education, understanding and motivation; and fostered by social and political policies and conditions. Almost all children attend school and spend 6-7 hours of their time everyday in that learning environment. Incorporating health into the school curriculum can have substantial influence on health promoting behaviours (HRIDAY PHFI report 2010).

1.1 Significance of the study: A comparative study on utilization of adolescent health services found that school based services were better utilized than health facility based services. The school health centers in the United States have adopted such approach by providing a broad range of reproductive services either on-site or by means of referrals. Promoting adolescent health and development by means of providing Adolescent Friendly Health Services requires complementary action by different stakeholders which are aimed at fulfilling their rights and addressing their special needs (Nath.A,, Garg.,S 2008). The results of the study will highlight the 
health promoting behaviours among adolescents after attending adolescent friendly services initiated at school campus through Multi Dimensional student life satisfaction scale.

Furthermore, a longitudinal study (Suldo \& Huebner 2002) showed that initial life satisfaction reports of adolescents moderated the relationship between their experiences of stressful life events and their later externalizing behavior. That is, adolescents with high levels of life satisfaction showed significantly less subsequent externalizing behavior in the face of adverse life events relative to students who were dissatisfied with their lives. Thus, life satisfaction appears to operate as an intrapersonal strength that helps buffer against the development of psychopathology in the face of increasing stressful life events. Taken together, research to date demonstrates that assessment of levels of life satisfaction in children and youth provide important information in and of itself, and also provides important information regarding risk for subsequent psychological problems.

The study done by Kotecha 2004 presents the findings as school based services are so much in demand and clinic based services are less utilized. Training teachers and parents is as much as important as counseling adolescent's issues. Ortabag 2011 et al., reported in a study that adolescent health promoting behaviors must be assessed in order to prepare effective school health programs. The study was done among adolescents in turkey and suggested that school environment provides important opportunities for health promotion and supporting the avoidance of risk behaviors. Ortabag 2011 et al., concluded, although high school age adolescents need more education and opportunities to focus on nutrition and exercise, health responsibility, stress management behaviors, all schools should consider it important, but they need to give higher priority to teaching and implementing specific programs that foster healthy lifestyles for adolescents. Risky behaviors in adolescents must be identified and be given more education and opportunities to develop more healthy lifestyles.

\subsection{Research Questions:}

The study aimed to examine the following the questions

1. What are the health and development problems prevailing among the young adolescents?

2. What are the health promoting behaviours of adolescents who attended adolescent friendly services at school?

3. Are guidance and counselling to adolescents on Physical health, psychosocial health, life style Modification and school performance through adolescent friendly centre be beneficial to adolescents?

1.3 Setting and Sample: The setting was a private higher secondary school at Coimbatore. The school authorities permitted the 9th and the 11th standard students to be included in the study. The age of adolescent's included in the study were from13-18 years of age. A total of 258 adolescents consisting of 101 boys and 158 girls, were included as samples. The word "students" are used synonymously for the term adolescents in this study.

1.4 Reliability of the tool: The MSLSS has internal consistency (alpha) coefficients have been reported in various publications, the findings suggest that the reliabilities all range from 70 to low 90 , thus they are acceptable for research purposes (Huebner 2001).

1.5 Data collection method: The data collection was done for a period of 9 months from June 2012-Feb-2013 in three phases.

1.5.1 Phase-1: In the first phase the socio demographic variables of the students were entered in the adolescent friendly center service admission form. A medical examination including the height, weight, BMI, dental, eye, ENT and a general examination was conducted for all the students. The students were asked to fill in the MSLSS scale, study skill checklist and the knowledge questionnaire (pretest) before the intervention. A structured teaching was delivered regarding the physical, psychosocial health and study techniques for boys and girls. Pamphlets were distributed to the students regarding adolescent nutrition, sample menu plan for adolescents, exercises and hygiene during adolescent period.

1.5.2. Phase-2: After 1 month the students had a guidance and counseling session on "Life skill training in adolescence". The students were individually approached and asked if they needed any guidance and counseling sessions. The students who were referred to the physician for the growth and developmental problems were assessed again, including all physical parameters.

1.5.3. Phase-3: After 45 days the third phase of the study was done to assess the effectiveness of the initiating adolescent friendly services in school. The post test was conducted on adolescent health. MSLSS tool was administered to students to test the effectiveness after intervention. The students were individually approached and asked if they needed any guidance and counseling sessions for any issues related to adolescent health, peer pressure, academic help or any specific issues. 


\subsection{Tools used in the study:}

1.6.1. Basic demographic data: Researcher filled in the adolescent friendly center admission form and collected the basic demographic data. Series of medical and physical examination was conducted including Height, weight, BMI, general physical examination, ENT , dental and eye ,to identify common problems.

1.6.2. MSLSS scale: The MSLSS provides the satisfaction of adolescents with important domains like school, family, friends, living environment and self, assess their general overall life satisfaction, demonstrate psychometric properties, and find out a replicable factor structure indicating the meaningfulness of the five dimensions. The 40 item MSLSS was administered individually to adolescents before and after intervention.

1.6.3 Structured teaching: A structured educational module containing adolescent growth and development, personal hygiene, nutritional needs of adolescents, importance of exercising, study tips, techniques to improve memory, solution to common problems during adolescents, life skills and study tips. The education was delivered in various phases of data collection. A questionnaire was developed to assess the pre and post education knowledge on above mentioned topics.

1.6.4. Study skill checklist: A study skill checklist developed by Virginia polytechnic institute and state University Blacksburg, VA 24061-0002 was used to assess the problems in study skills of adolescents. This checklist consists of 21 items in various areas including reading habits, writing skills, time scheduling, concentration, listening and note taking and testing / exams.

\section{Main findings of the study:}

The findings as discussed as per the objectives of the study.

2.1. Baseline sample characteristics of participants who attended the adolescent friendly services: The mean age of adolescents is 14.5years. Majority of the samples (61\%) of them are adolescent girls and 39\% of the samples are boys. The mean height of boys is $165.65 \mathrm{cms}$ and mean height of girls is $157.53 \mathrm{cms}$. The mean weight of the boys is $54.56 \mathrm{kgs}$ and the mean weight of girls is $52.08 \mathrm{kgs}$. Majority of the adolescents girls $51.89 \%$ and $47.52 \%$ of boys are in the (healthy weight) BMI range of 18.5-24.9. Unfortunately a significant proportion of the adolescent girls $30.37 \%$ and $39.60 \%$ of the boys are found to be underweight, below the 5th percentile. Minimal percentage of the boys and girls are obese. The menstrual history of adolescent girls shows that $93.67 \%$ of the girls have attained puberty and $6.32 \%$ of adolescent girls did not attain puberty. Regarding the academic grade of adolescents majority of them were in the "B" grade. The main leisure time activity of adolescents is watching television. Majority of adolescent boys (48) and girls (78) have not taken leave to school. A very few adolescents avail leave from school. The reason for being absent to school varied according to the situation. Regarding the dietary pattern of adolescents, it showed majority of girls (62) and boys (42) skip breakfast. A minimal number of adolescent girls (24) and boys (12) boys eat fast food daily. The physical activity pattern of adolescents showed that majority of the girls (112) engage only in school sports activities. While among boys 40 of them involve in school sports and equal amount of boys also involve in daily exercising in some form like karate, gym etc.

2.2. To identify the types of health and developmental problems prevailing among the adolescent group: The physical examination findings revealed the following results. Dental carries was the most common finding among boys (10.89\%) and girls (14.55\%). Newly identified refractive errors were found to be $5.9 \%$ in boys and $10.5 \%$ among girls. The other common physical examination finding among girls and boys were use of glasses for refractive errors are $25.74 \%$ among boys and lesser in girls $10.75 \%$.

2.3. Study skill check list: Majority of the adolescents $(57.91 \%)$ had problems in writing skills. Next to that was time scheduling (44.01\%),encountering exams (34.36\%), difficulty in concentrating (30.88\%), and reading the day before exams $(30.11 \%)$ problems in daily reading $(27.79 \%)$ and the least encountered problem was listening and note taking $(24.32 \%)$.

2.4. To identify the changes in health promoting behavior among adolescents attending adolescent friendly center services at school: To measure the health promoting behavior of adolescents after attending the adolescent friendly center the Multi Dimensional Student life Satisfaction scale was used.

2.5. Multi Dimensional Students life Satisfaction Scale -Assessment of Boys: Paired ' $t$ ' test results revealed that it is significant at 0.01 level indicating that the two components, "school" and "self" has a significant difference in the mean scores of the boys, obtained before and after the initiation of the Adolescent Friendly Center Service at school.

\subsection{Multi Dimensional Students life Satisfaction Scale - Assessment of girls:}

Paired ' $t$ ' test results revealed that it is significant at $\leq 0.01$ level indicating that the component "self" as a significant difference in the mean scores of the girls, obtained before and after the initiation of the Adolescent Friendly Center Service at school. 
2.7. Multi Dimensional Students life Satisfaction Scale: Overall Assessment results reveal the component "self" has a significant difference in the mean scores of the adolescents, obtained before and after the initiation of the Adolescent Friendly Center Service at school. The t value is 3.484 and is significant at .001level.

2.8. To provide guidance and counselling to adolescents on Physical health, psychosocial health and life style Modification and school performance through adolescent friendly centre: The adolescents were provided with structured teaching on various adolescent issues, life skill training and study techniques.

2.9. Analysis on the knowledge scores of adolescents before and after intervention: The results of the study reveal that the knowledge scores aspects in each individual component (on adolescent issues like adolescent age, required caloric need for adolescents, the term anemia, the term obesity, the term anorexia, pre menstrual symptoms, menstrual problems, dysmenorrhoea, cause for anemia in adolescent girls, the term contraception, )has a significant difference in the mean scores of the adolescents at 0.01 level, obtained before and after the initiation of the Adolescent Friendly Center Service at school. This shows that the structured teaching and guidance and counselling sessions were effective.

2.10. Analysis on the overall knowledge scores of adolescents before and after intervention: The results of the study reveal that the overall knowledge score (on adolescent health and issues) has a significant difference in the mean scores of the adolescents at 0.01 levels, obtained before and after the initiation of the Adolescent Friendly Center Service at school.

2.11. Feedback on adolescent friendly services by adolescents: Majority of adolescent boys $(80.19 \%)$ and girls (87.34\%) felt that information on reproductive health and physical health screening was very helpful to them.75.24\% of adolescent boys and 53.64\% of girls felt the guidance and counseling services were beneficial. $55.44 \%$ of adolescent boys and $51.89 \%$ of girls felt that adolescent friendly services created self awareness among them. Academic help including study skill checklist helped them to find out their study problems and techniques to improve studies were found to be beneficial for $60.39 \%$ of adolescent boys and $70.25 \%$ of girls.

\section{Discussion}

Adolescent health is not a main stream strategic component of health care in India. Of late adolescent health has been included as a component of the reproductive health package in the RCH services program. However, there is no clear definition of strategic approach and activities to provide adolescent health care (ARH India report 2003). Hence a school based adolescent friendly service was initiated at the school campus by the researcher.

A study by Karki 2004 on young people who were attending adolescent friendly services at Kathmandu Nepal reported that Adolescents are the special group of population whose needs should be sensitively addressed. Adolescent friendly centre can play a vital role to support and help a good number of adolescents of the society, need of a separate adolescent friendly centre or a separate department of adolescent medicine in all health institutions and other regions of the country seem to be very clear. Therefore number of such centers should be increased but the environment of such centre should be suitable to cater both the sexes of adolescents equally. Adolescents need to be provided with adequate information and skill, counseled and supported for continuity of their education and get married at an age when they are able to lead a dignified independent life (Karki 2004).

The integration of positive as well as negative indicators of well-being into health assessment yields a more comprehensive picture of functioning. Furthermore, understanding how mental and physical wellness relate in a holistic manner allows professionals to examine the health and behavior of youth as a set of integrated factors rather than isolated pieces. Such data can be used to inform wellness programs and prevention strategies targeting children and adolescents who may be at-risk of developing future health problems - those who are not displaying symptoms of physical or mental illness, but who also report decreased life satisfaction, or happiness, and low amounts of health-promoting behaviors such as regular exercise and healthy diet. By examining these types of wellness components in youth, practitioners and researchers could better understand and address the health concerns of this population via a prevention model of service delivery (Hudkins 2011). For most adolescents, school is the most important setting outside the family. Student's perceptions of the school environment are associated significantly to their health and well being (Eccles 1993). Perceived high-level supports from students are related to lower subjective health complaints, satisfaction with school, and increased physical activity (Samdal 1998).

The results of the present study reveal that the component of the MSLSS scale "self and" school" has a significant (at 0.01 levels) difference in the mean scores of the adolescents, obtained before and after the initiation of the Adolescent Friendly Center Service at school. The results of the study further shows that the knowledge score (on various adolescent health issues) in each individual component has a significant difference in the mean scores of the adolescents, obtained before and after the initiation of the Adolescent Friendly Center Service at school. These results prove that the adolescent friendly services using school based approach is beneficial to the adolescents in promoting their health related behavior. 
3.1. Limitation of the study: Since the study was done inside school campus the teachers and parents were more towards academic help to their children than to learn about issues of adolescent health, especially sexual and reproductive health, substance abuse etc. It was challenging to get their concern before talking to adolescents on the specified issues.

3.2. Recommendations: Training can be provided to school teachers, community leaders and multipurpose health workers regarding adolescent friendly services and ideal way of providing guidance and counseling services. The school authorities can be requested to approach NGOs for continuing adolescent friendly services at school campus.

\subsection{Conflicts of interest: none}

3.4. Conclusion: In conclusion this study clearly shows that initiating adolescent friendly services in school is beneficial for the adolescents. The study results show that 2 (self and school) domains of Multi Dimensional Student Life Satisfaction Scale has significant difference at 0.01 level before and after attending the services at school campus. These types of services can bring about changes in health promoting behaviour of adolescents. Careful planning and execution of school based adolescent friendly services in collaboration with adolescents, parents, teachers and local community is required. These types of services should only be used as complementary services and should not replace other community health provisions. Further studies can begin to define minimum standards for comprehensive adolescent friendly health services and can ascertain "what is required' using appropriate research methodology.

3.5 Implications for future practice: This study findings provide important guidance for parents, teachers, school authorities and local community that adolescents wish to have adolescent friendly services through school based approach. These findings also suggest adolescent views, expectations and fears should be assessed in consultation to appropriately to respond to them. Develop interventions to incorporate involvement of parents and teachers to be educated and be able to change their perceptions and attitude about adolescent health involving reproductive child health and show willingness to dialogue with their children.

\section{Funding and Acknowledgements:}

No funds were requested.

4.1. Ethical considerations: Full ethical approval was obtained from the university vetting committee, local school authorities, written consent from adolescents, parents and teachers for their willingness to participate in study.

\section{References:}

[1] WHO (2008.) The millennium development Goals report. Facts on Adolescents

[2] Viner R, Barker M (2005). Young people's health: the need for action. BMJ.330 (7496) PMC 556170.

[3] Raj S, Senjam SS, Singh A. Assessment of health-promoting behavior and lifestyle of adolescents of a north indian city. Int J Prev Med 2013;4:1189-93.

[4] Framework criteria for healthy school seal of approval. An initiative by AROGYA world in collaboration with HRIDAY and PHFI 2010 .

[5] Nath A, Garg S (2008). "Adolescent friendly health services in India: A need of the hour," Indian Journal of Medical Sciences. 62(11) 465-72

[6] Huebner, E. S., Suldo, S. M., Valois, R. F., \& Drane, J. W (2002a). Brief Multidimensional Students' Life Satisfaction Scale (BMSLSS): Normative data for middle school students. Manuscript submitted for review.

[7] Huebner, E. S., Suldo, S. M., Valois, R. F., Drane, J. W., \& Zullig, K. (2002b). Brief Multidimensional Students' Life Satisfaction Scale (BMSLSS): Gender, race, and grade effects. Manuscript submitted for publication.

[8] kotecha.p.v (2004). Adolescents friendly health service model proceedings of 31st annual national conference of indian association of preventive and social medicine, 27th to 29th feb., 2004, pgimer, chandigarh, indian journal of community medicine 29 , no. 5

[9] Ortabag T, Ozdemir S, Bakir B, Tosun N (2011).Health promotion and risk behaviors among adolescents in Turkey.The Journal of School Nursing (Impact Factor: 0.69). 05/2011; 27(4):304-15. DOI:10.1177/1059840511408322.

[10] Huebner, E.S (2001). Manual for the Multidimensional Students' life Satisfaction Scale. Columbia: University of South Carolina. Document Number).

[11] ARH India 2003 report. National Youth policy - India (2003).

[12] Karki C (2004). "Study of young people attending an adolescent friendly centre",. Kathmandu University Medical Journal (KUMJ).;2(4) .324-30.

[13] Hudkins,EJS (2011). Health Promoting Behavior And Subjective Well Being Among Early Adolescents. A Dissertation Submitted In Partial Fulfillment Of The Requirements For The Doctor Of Philosophy, Department Of Psychological And Social Foundations, College Of Education, University Of South Florida.

[14] Eccles J, et al (1983). Development during Adolescence: the impact of stage- environment fit on young adolescents' experiences in schools and families. Am Psychology. 48: 90-101.

[15] Samdal O, Dur,W (1998). The school Environment and the health of adolescents: WHO EMRO Report. 